Migrations, résidence et représentations de la famille : les familles caribéennes en France et en Grande-Bretagne

Stéphanie Condon et Margaret Byron

\author{
(2) OpenEdition \\ Journals \\ Édition électronique \\ URL : https://journals.openedition.org/remi/4247 \\ DOI : $10.4000 /$ remi.4247 \\ ISSN : $1777-5418$ \\ Éditeur \\ Université de Poitiers \\ Édition imprimée \\ Date de publication : 1 avril 2008 \\ Pagination : 35-63 \\ ISBN : 978-2-911-627-48-2 \\ ISSN : 0765-0752
}

Référence électronique

Stéphanie Condon et Margaret Byron, « Migrations, résidence et représentations de la famille : les familles caribéennes en France et en Grande-Bretagne », Revue européenne des migrations internationales [En ligne], vol. 24 - $n^{\circ} 1$ | 2008, mis en ligne le 01 avril 2011, consulté le 14 avril 2022. URL : http://journals.openedition.org/remi/4247 ; DOI : https://doi.org/10.4000/remi.4247

Ce document a été généré automatiquement le 14 avril 2022.

(c) Université de Poitiers 


\title{
Migrations, résidence et représentations de la famille : les familles caribéennes en France et en Grande-Bretagne
}

\author{
Stéphanie Condon et Margaret Byron
}

Les auteures remercient Cédric Audebert ainsi que le comité de rédaction pour leurs précieuses remarques sur la première version de cet article.

1 La famille caribéenne a longtemps fasciné les sociologues et les anthropologues nordaméricains et britanniques. Entre les années quarante et soixante-dix, le contexte de la reproduction a été un objet central des recherches et l'on a tenté d'expliquer l'instabilité des couples, soit par le maintien des structures polygames africaines, soit comme conséquence de l'esclavage (Frazier, 1940 ; Barrow, 1996 ; Charbit, 1987 ; Smith, 2001). De forts taux de naissances illégitimes, des unions instables et des proportions élevées de ménages dirigés par des femmes ont été interprétés comme les traits des "classes inférieures désorganisées" (Smith, 1988). Malgré des références fréquentes aux liens forts entre les membres féminins de la parenté et au mode d'organisation des ménages « matrifocaux », le fait que ce «système familial » soit diamétralement opposé au « modèle bourgeois » - reconnu comme la norme universelle de stabilité familiale et sociale - a conduit à ce que ces formes de famille soient considérées comme des structures inappropriées pour l'éducation des enfants (Gautier, 1993). Les études des familles antillaises en Grande-Bretagne à partir des années soixante et les discours qui prévalent dans les institutions et dans les services sociaux britanniques et français se sont appuyés - et s'appuient souvent encore - sur de telles présuppositions. Si des recherches récentes ont apporté un regard plus approfondi sur le "système familial caribéen » (Attias-Donfut et Lapierre, 1998 ; Byron, 1998 ; Charbit, 1987 ; Goulbourne et Chamberlain, 2001), les représentations stigmatisantes, qui instruisent une large littérature scientifique et les discours institutionnels dans plusieurs sphères, sont difficilement ébranlées et contribuent à une racialisation de ces populations. 
2 L'étude de la famille a souvent été limitée par la confusion entre "famille " et «ménage ». La superposition des deux concepts a conduit à ce que les liens entre les membres d'un ménage et la parenté en dehors de ce ménage, et le rôle de ces derniers dans l'organisation sociale du réseau familial soient ignorés (Solien, 1960; Barrow, 1996 ; Hareven, 2000). Tamara Hareven a souligné le rôle joué par les historiens dans cette déformation de la famille : «Par mégarde, la recherche historique a contribué au mythe de la famille nucléaire isolée prévalant dans les théories sociologiques » (2000: 32). On peut en dire autant des écrits des années quarante et cinquante sur la famille caribéenne, où les liens familiaux au-delà du ménage, si importants dans ces sociétés, étaient laissés de côté (Solien, 1960 ; Barrow, 1996). Dans les études qui s'appuient sur des données statistiques, la notion de la famille a généralement coïncidé avec celle du ménage; tendance qui se perpétue aujourd'hui, par exemple dans les analyses de la famille caribéenne en Grande-Bretagne à partir du recensement (Owen, 2001) où le terme famille monoparentale est utilisé pour décrire les ménages dans lesquels une mère vit seule avec un ou plusieurs enfants. Ainsi, les liens forts qui existent entre deux ménages ou plus qui, aux yeux de leurs membres, constituent «la famille» sont ignorés. Ces groupes familiaux s'étendent à travers plusieurs générations et sur des distances souvent importantes. De même, au début d'un courant migratoire, un seul ménage peut comporter plusieurs "familles nucléaires ». Les liens familiaux au-delà des frontières sont maintenant mis en avant dans la littérature sur «les familles transnationales" (Bryceson and Vuorella, 2002 ; Goulbourne, 1999; Olwig, 2001; Vertovec, 2003) et la distinction entre famille et ménage est centrale dans cette perspective.

3 La comparaison entre les contextes français et britannique des migrations caribéennes est très éclairante sur ces différentes représentations de la famille caribéenne et leur impact sur la vie quotidienne des migrants caribéens et de leurs descendants dans les deux métropoles européennes. Au niveau du ménage, les caractéristiques des deux populations sont très proches dans la mesure où les mères seules vivant avec enfants sont proportionnellement plus nombreuses que dans l'ensemble de la population, seul indicateur disponible d'un système familial non centré sur le couple. Si un examen plus approfondi révèle d'autres facteurs que culturels, c'est-à-dire le rôle des normes au sein d'un groupe social (Charbit, 1987b), on a tendance à se focaliser sur ce qui paraît « anormal » et à se satisfaire de ces apparences pour tenir à distance une population. D’autres perspectives méthodologiques sont nécessaires pour resituer ces ménages dans leurs réseaux familiaux; mais jusqu'à présent elles s'imposent difficilement comme mode de lecture - d'une lecture nouvelle - des familles caribéennes. Les catégorisations employées dans les deux contextes, et les représentations qui les alimentent, ont pesé sur le devenir de ces populations; le regard stéréotypé sur la famille caribéenne a été un élément important dans ces processus de catégorisation. L'étude de l'accès au logement est très révélatrice de ces processus et de l'interaction entre divers rapports sociaux qui s'inscrivent dans l'héritage colonial et post-colonial (Condon, 1995) : la localisation dans les grandes villes, étroitement liée au recrutement des travailleurs migrants ; les attentes des migrants vis-à-vis de l'État pour leur assurer un logement convenable; les discriminations vécues au cours de la recherche d'un logement. Malgré des histoires migratoires différentes, les populations caribéennes en France et en Grande-Bretagne affichent de grandes similitudes en matière de résidence, tout comme en terme d'insertion professionnelle, et ces similitudes sont le reflet d'une expérience commune au sein de l'émigration caribéenne. 


\section{Les formes familiales caribÉennes : « instabilité et absence de structure » ou « solidaritÉs familiales et flexibilités "?}

4 La fascination du monde académique anglo-américain devant la "famille caribéenne » provient de l'identification d'un "modèle caribéen» de la famille qui serait diamétralement opposé au "modèle bourgeois »; c'est-à-dire, à la famille nucléaire, patriarcale. L'instabilité du couple, l'absence du conjoint ou du père des ménages, de forts taux de naissances illégitimes sont les caractéristiques reconnues de ce modèle caribéen. La perspective de chercheurs comme Simey, 1946; Clarke, 1957 ; Davison, 1966 - même si elle était rarement explicitée comme telle (Phoenix, 1993) - était celle de la famille nucléaire comme forme de famille normale, idéale pour la bonne éducation des enfants et celle de la nécessité de la présence du père comme élément stabilisateur dans le fonctionnement du ménage (Barrow, 1996). Ces auteurs ont eu tendance à laisser de côté le dynamisme des familles et la variété des formes observées pour ne se focaliser que sur l'instabilité de la relation de couple et les ménages «sans mari, sans père ».

5 Une deuxième dimension du modèle largement reconnue est l'importance de la matrifocalité des relations intra-familiales. On a souvent parlé d'un système "matriarcal», ce qui suppose une domination par les femmes de l'organisation familiale. Une analyse plus générale des relations hommes-femmes nuance cette vision d'une domination féminine, en soulignant les normes de comportement différentes pour les hommes et les femmes; d'une part, la respectabilité pour les femmes, d'autre part, la réputation pour les hommes (Wilson, 1973). La recherche de moyens de gagner et de maintenir une réputation éloignerait les hommes du foyer, tandis que la femme gagnerait le respect en se comportant en fille et/ou mère exemplaire. Plusieurs auteurs ont approfondi cette analyse des rapports de genre dans les sociétés caribéennes dans le but de mieux comprendre l'instabilité du couple, les inégalités sociales, l'enfermement des hommes et des femmes dans des rôles sexués très rigides ${ }^{1}$. La perspective de Smith sur la famille matrifocale a été de souligner que, plutôt qu'une domination par les femmes en tant que "chefs de ménage ", ce serait les femmes, en tant que mères, qui seraient au centre des relations familiales. Si ce modèle n'est pas généralisable à tous les milieux dans la région, ni à toutes les époques, la position sociale reconnue des femmes au sein des réseaux familiaux, leurs rôles dans le système d'échanges, de dons, d'obligations constitue un concept clé dans l'analyse des migrations et des réseaux familiaux transnationaux.

6 Ce qui a manqué dans le débat autour de la famille caribéenne dans les années 1950-70 est la prise en compte de la variété des formes familiales et l'importance des paramètres tels que la classe sociale, le genre, l'ethnicité, l'âge du chef de ménage. Les études se sont focalisées largement sur la population des descendants d'esclaves africains, la majorité de la population aux Antilles, dont seule une petite partie avait accédé alors aux classes moyennes (fonctionnaires, professions libérales, commerçants). Cependant, d'autres systèmes familiaux coexistaient dans les îles caribéennes; non seulement ceux des colons mais aussi ceux des descendants des travailleurs indiens recrutés sur contrat à la fin de l'esclavage. Le système familial de ces populations correspond à un modèle de la famille étendue unissant deux familles, 
unité de base dans les stratégies familiales et dont l'élément central est le mariage. Les analyses des familles indo-caribéennes réalisées par Reddock et al. (1999) et Smith (1996) ont souligné l'importance de l'ethnicité, et les évolutions au cours du temps, pour comprendre les configurations familiales. De même, prendre en considération la dimension du temps permet de mettre en évidence la dynamique des ménages : comme l'ont démontré Charbit et Léridon (1980), si la majorité des femmes adultes passe une période de leur vie dans un ménage monoparental, à un moment donné, ce type de ménage ne représente pas la forme majoritaire dans ces sociétés.

7 Les analyses de la famille aux Caraïbes qui laissent de côté la dimension de classe auraient tendance à accentuer le processus de racialisation de ces populations. En n'étudiant que les familles caribéennes des "classes populaires", les chercheurs ont ignoré les relations de couple - et donc une partie des dynamiques de la fécondité dans les classes moyennes ou bourgeoises. C'est Raymond Smith $(1988,1996)$ qui, en orientant son regard vers les comportements masculins dans ces classes plus aisées au milieu du vingtième siècle, a observé qu'il était courant que les hommes mariés, chefs de "famille nucléaire», aient des relations intimes avec des femmes des classes populaires. Les normes de leur groupe social interdisaient les unions formelles avec des personnes des classes populaires; et donc les règles de cette hiérarchie sociale ont encouragé la prolifération des relations « informelles » et un système où les hommes avaient deux partenaires et, le plus souvent, des enfants « dehors ». Cette pratique était très révélatrice des rapports de classe et de race aux Antilles, la deuxième femme issue des classes populaires étant maintenue dans un statut inférieur, dépendant. Ce serait également l'héritage de la première période des sociétés coloniales, une époque où le concubinage ou des liaisons de courte durée étaient préférés au mariage par les colons masculins (Burnard, 2006). Les comportements de ces générations d'hommes à la Jamaïque, qui ont suscité de vives réactions moralisantes en métropole et des craintes pour l'avenir de la colonie, ont fait coulé beaucoup d'encre à l'époque (idem). Cependant, ce passé et le rôle des colons dans cette "culture d'instabilité conjugale " semblent avoir été oubliés dans les années 1940-60 au moment des études de la famille dans les milieux populaires. À cette époque, le modèle bourgeois de la famille nucléaire s'était répandu dans les classes moyennes et le mariage mis en avant comme symbole de respectabilité. En prenant le niveau de scolarisation comme indicateur de classe sociale, Charbit et Leridon (1980) ont observé que le mariage était le plus répandu dans les couches les plus scolarisées et s'était imposé donc face à l'union libre.

La vision plutôt négative de l'organisation des familles caribéennes descendantes d'esclaves a produit les images stéréotypées de la femme - ou de la mère - caribéenne. Dans ces représentations, on retrouve deux stéréotypes forts : l'un qui semble valoriser la femme, l'autre qui la stigmatise. Mais les deux lui assignent un statut de dépendante. D'un côté, on retrouve l'image de la mère courageuse, résiliente, devant subvenir aux besoins matériels de la famille et assumer seule l'éducation des enfants; de l'autre, celle de la mère assistée, vivant d'allocations de mère isolée ou d'autres prestations sociales, parvenant à surveiller ses enfants tant bien que mal. En parallèle, et toujours construit par opposition à une norme silencieuse, l'homme caribéen est à la fois une "présence pathologisée » (Phoenix et Woollett, 1991), caractérisé par sa supposée sexualité débridée ou par la nature violente qui lui est attribuée; et une absence du foyer et du monde du travail. En même temps il est représenté comme victime et acteur de ces absences, conséquences du sous-emploi et du chômage, de l'alcoolisme et de l'instabilité conjugale. Ce sont ces mêmes stéréotypes que l'on retrouve dans des 
discours institutionnels et dans certaines littératures scientifiques traitant des populations « noires » aux États-Unis (Hill Collins, 1994) ou en Afrique du Sud (Macleod et Durrheim, 2002). Et ces représentations de familles "désorganisées » façonnent le regard d'acteurs institutionnels et de chercheurs sur les migrants caribéens qui arrivent dans les villes européennes à partir des années 1950.

Dans les métropoles européennes, la vision stéréotypée des relations de couple et de la famille caribéenne persiste. Certains iront jusqu'à suggérer que la migration ne ferait qu'exacerber le problème de « désorganisation » dans les familles (Davison, 1966), une perspective qui ignore les réseaux de parenté fortement structurés qui permettent et maintiennent les courants migratoires caribéens (Philpott, 1977 ; Byron, 1994 ; Olwig, 2001 ; Byron et Condon, 2008). Dans le travail social auprès de ces populations, les interprétations hégémoniques, coloniales des sociétés caribéennes et de l'absence de la famille nucléaire ont longtemps perduré (Pryce, 1979) et influencé les discours institutionnels, depuis les acteurs du logement social jusqu'au système éducatif. Or ce sont justement la souplesse et le caractère robuste des réseaux familiaux, non pris en considération dans les études de la famille caribéenne, qui ont non seulement permis la migration vers les métropoles européennes mais aussi aidé à contourner les nombreux obstacles posés par les attitudes racistes et les discriminations pratiquées par les institutions. Par ailleurs, certains proposent qu'au-delà de ces rôles de soutien, la famille caribéenne constitue une forme familiale pionnière, moderne, qui anticipe les aspects de la vie familiale qui se généraliseront dans un avenir proche (Thompson et Bauer, 2005 : 21).

\section{Famille et résidence dans deux contextes de migrations (post-)coloniales}

10 La composition des ménages, les configurations familiales et les caractéristiques résidentielles des populations d'origine caribéenne en France et en Grande-Bretagne ont été fortement influencées par l'évolution des migrations, le rôle de l'État dans l'accueil des migrants et l'insertion professionnelle des individus. Un rappel du contexte de l'installation de ces populations dans les deux métropoles est donc utile.

\section{Contextes politiques et économiques de l'émigration caribéenne}

11 Depuis la première moitié du XIX ${ }^{e}$ siècle, et la libération des esclaves, l'espace caribéen a connu une nouvelle dimension migratoire (Domenach, 2002). Dans cette région, jusqu'alors espace d'immigration, une pratique migratoire s'est développée entre les îles, surtout à partir des petites îles vers les grandes ou vers le continent sud-américain, traversant souvent des barrières linguistiques (Carnegie, 1987; Patterson, 1978; Thomas-Hope, 1986). Au début du XXe siècle, des milliers d'hommes migrants sont partis travailler dans la construction du Canal du Panama, et l'exploitation du pétrole en a amené d'autres au Venezuela ou dans les raffineries des îles néerlandaises. Puis, vers 1930, après l'arrêt définitif de ces émigrations et de l'immigration contractuelle venue notamment d'Asie, les populations caribéennes sont entrées dans une phase de stabilisation et de croissance naturelle, ceci pour la première fois dans leur histoire. Avec la baisse de la mortalité, grâce aux progrès médicaux et sanitaires, s'est amorcée l'explosion démographique. C'est cette pression démographique qui est le plus souvent 
citée comme étant l'un des facteurs des flux d'émigration massifs, les autres facteurs étant le manque de diversification des économies des îles, la misère, le chômage, le sous-emploi. Cependant, comme l'a démontré Ceri Peach (1968), ce sont des facteurs passifs et non pas dynamiques : ils permettent à l'émigration de se produire, mais ne la provoquent pas directement. En effet, l'amorce a été l'appel à la main-d'œuvre de la part des pays industrialisés, les anciens colonisateurs de ces îles (Brock, 1986; Byron et Condon, 1996, 2008). Comme nous le verrons, cet appel s'est opéré de manière différente en France et en Grande-Bretagne.

$\mathrm{Au}$ lendemain de la Seconde Guerre mondiale, les deux contextes français et britannique présentaient de fortes similarités dans le rapport métropole - colonies antillaises. Dans un projet de maintien sur le plan mondial des espaces politiques et culturels des deux États, la citoyenneté - sous des formes tout de même différentes - a été accordée aux habitants de ces colonies (Murch, 1971 ; Brock, 1986). Or au cours des années suivantes (la période de décolonisation qui a concerné la plupart des îles caribéennes), le lien politique entre les divers territoires - métropoles et colonies s'est transformé et, par la même occasion, le statut politique des migrants aussi. Ainsi le statut des Jamaïcains partant pour la Grande-Bretagne avant l'indépendance de l'île était assez semblable à celui des migrants martiniquais ; par la suite les statuts allaient diverger.

La politique d'assimilation mise en œuvre par l'État français, et renforcée à partir de la départementalisation en 1946, visait à intégrer les populations des îles de la Réunion, de la Martinique et de la Guadeloupe et aussi celle de la Guyane dans la nation française. Les diverses institutions ont étendu leur champ d'exercice jusqu'à ces nouveaux départements. D'une certaine manière, c'était un approfondissement des mécanismes déjà en place depuis la fin de l'esclavage (administration, école...) ; mais à partir des années cinquante, l'État est intervenu de manière plus active dans tous les domaines, y compris celui de la fécondité (Charbit et Leridon, 1980). La gestion du domaine économique s'est faite cependant avec plus de difficulté. Comme la plupart des économies insulaires de la région, les économies des Antilles traversaient alors une crise profonde causée par l'accroissement de la concurrence en terme de production de sucre. En l'absence de production économique de substitution, la pression sur le marché du travail de ces îles, limitées par leur superficie, s'est renforcée. En même temps, le développement excessif du secteur tertiaire administratif public, octroyant des salaires très avantageux, a creusé un fossé de plus en plus profond entre les nantis et les petits revenus (Domenach et Picouet, 1992 : 112-114). Cette situation a rendu particulièrement favorable la constitution d'un potentiel migratoire, potentiel que l'État central allait pouvoir capter et canaliser dans une politique plus globale. Dès 1954, l'émigration de nombreux habitants des îles de la Martinique et de la Guadeloupe était jugée indispensable comme solution au surpeuplement de ces départements. Les problèmes de chômage et de sous-emploi, ainsi que les troubles sociaux liés à la fermeture des usines sucrières et les manifestations de mécontentement vis-à-vis du statut départemental de ces îles ${ }^{2}$ (comme à Fort-de-France en 1959), étaient considérés comme tributaires de ce surpeuplement. C'est ainsi qu'à la fin des années cinquante, une véritable politique d'émigration a été élaborée, puis mise en œuvre à partir de 1962-63 grâce à la création du Bumidom, agence d'état responsable de l'organisation du départ vers la métropole de travailleurs antillais ou réunionnais. Le but initial était de faire partir entre 6000 et 7000 personnes des Antilles françaises chaque année et 
d'encourager l'émigration de personnes des deux sexes et de familles, afin d'éviter un déséquilibre démographique qui résulterait du seul départ d'hommes adultes (CGP, 1959 : 407-408). Ce rythme des départs a été encouragé jusqu'au milieu des années 1970, puis la crise économique a eu pour conséquence une réorientation de la politique vis-àvis des migrations en provenance des DOM (Constant, 1987). En tout cas, l'objectif a été largement atteint puisque le Bumidom a organisé le voyage, l'accueil et le placement de 85863 individus, dont le placement de 11928 militaires du contingent démobilisés en métropole (Condon et Ogden, 1991) et ces migrations organisées ont entrainé le départ d'un nombre croissant de migrants « spontanés ».

Si les îles anglaises étaient confrontées à une situation très proche, l'État britannique n'a pas joué de rôle décisionnel analogue à celui observé dans le contexte français dans la résolution de problèmes socio-politiques dans les Caraïbes; c'était aux gouvernements locaux de mener leurs propres plans d'action. Seule la relation entre Porto Rico et les États-Unis se rapproche de celle entre les Antilles et la France (Milia, 2002). Citoyens britanniques et du Commonwealth à partir de 1948, les habitants de ces îles bénéficiaient du droit d'entrée en Grande-Bretagne dans le but d'y travailler, d'y résider et d'y voter. Une migration spontanée avait démarré dès le passage de la loi de 1948 avec l'arrivée de 417 migrants jamaïcains sur le bateau Empire Windrush, puis le retour de nombreux anciens engagés de l'armée de l'air. À la différence du cas français, il n'y a pas eu de politique de recrutement envers les populations caribéennes. De forts besoins en main-d'œuvre peu qualifiée ont poussé l'État britannique à organiser le recrutement de travailleurs irlandais ou de «travailleurs volontaires européens" (dans le cadre d'un programme concernant les personnes déplacées en Europe suite à la Seconde Guerre mondiale). Cependant, à l'invitation du gouvernement de la Barbade, île dont la densité démographique était particulièrement forte, le gouvernement britannique a accepté de mettre en place une opération de recrutement. Plusieurs entreprises nationalisées dans le secteur des transports et des hôpitaux, et aussi quelques grands hôtels et restaurants, ont coopéré avec le gouvernement de la Barbade pour recruter et financer le voyage des travailleurs. Un bureau à Londres a supervisé l'opération et le placement des migrants. Comme dans le cas français, ces populations ont été attirées par l'opportunité de travailler dans les services publics britanniques, y voyant un chemin vers la promotion sociale. Entre 1955 et 1961, 4404 migrants, hommes et femmes, ont bénéficié du programme. Mis à part ces quelques initiatives de recrutement à la Barbade, la plupart des migrants sont partis de façon spontanée et sans encouragement de la part de l'État britannique ; au contraire, ces gouvernements très frileux ${ }^{3}$ redoutaient les réactions de la population native des îles britanniques.

$\mathrm{Au}$ moment où l'État français mettait en œuvre sa politique d'émigration des Antilles, par l'intermédiaire du Bumidom, une Loi sur l'immigration du Commonwealth a été votée au parlement britannique. Cette loi résultant d'un débat politique de longue date a entériné la volonté d'un groupe d'acteurs politiques qui luttaient contre l'immigration de personnes dont les caractéristiques « raciales et religieuses » n'étaient pas considérées comme conformes à celles de la majorité britannique (Carter et al., 1987). À partir de juillet 1962, seuls les dépendants des migrants résidant en GrandeBretagne avant cette date ont eu le droit d'entrée. Toute autre personne souhaitant s'installer dans le pays devait faire la demande d'un permis de travail. L'évolution des effectifs d'immigrants au cours des quelques années précédant la loi témoignent de la diffusion d'informations dans les colonies sur l'imminence de cette loi: environ 20000 Antillais sont arrivés en 1958, 50000 en 1960 et plus de 60000 en 1961 
(Annexe 1). Une grande partie de ces effectifs était composée de dépendants mineurs ou âgés, leur départ étant précipité par la peur d'être séparés durablement des autres membres de la famille et de manquer l'occasion de s'installer en Grande-Bretagne (Peach, 1968). Par la suite, le nombre d'entrées a chuté, variant entre 8000 et 15000 par an entre 1963 et 1967 et devenant négligeable à partir de 1969. C'est aussi à partir de 1970-1971 que les sorties antillaises du territoire britannique ont dépassé les entrées.

16 À la différence des premières migrations de travail dans la région, dominées par les hommes, la migration vers les métropoles européennes s'est rapidement féminisée. Dans le cas britannique, ce sont les épouses ou compagnes des hommes partis travailler dans l'industrie ou dans le secteur des services (transports publics, hôtellerie...) qui les ont rejoint dans les années 1950. Certains de ces hommes retournaient en GrandeBretagne après avoir servi dans les armées ou avoir travaillé dans les usines de guerre. Puis d'autres femmes ont quitté les îles anglophones, en dehors du cadre d'un regroupement familial, dans le but d'exercer le métier d'infirmière. Ce souhait a été satisfait pour de nombreuses femmes, tandis que d'autres ont trouvé d'abord un emploi d'agent hospitalier, d'employée dans l'hôtellerie ou la restauration ou d'ouvrière dans le textile. Les besoins des entreprises britanniques ayant primé sur les préférences des gouvernements de ce pays, le nombre d'hommes et femmes quittant les îles des Caraïbes pour y travailler s'est accru rapidement. Au recensement de 1961, on dénombrait 75992 femmes et 95804 hommes migrants caribéens résidant en GrandeBretagne (Annexe 1).

17 En France, vers le milieu des années 1950, de nombreux hommes antillais, peu qualifiés, ont été recrutés par les administrations, notamment par la Police, les Douanes et les Postes et Télécommunications. Puis, à partir de 1960, la conscription a été introduite dans les DOM. Environ la moitié du contingent né aux Antilles devait effectuer son service militaire en métropole, l'un des buts de l'État étant d'établir une nouvelle voie d'émigration ${ }^{4}$. Une formation professionnelle a été offerte aux conscrits démobilisés en métropole, ainsi que la possibilité de garder leur billet de retour aux Antilles pendant cinq ans (Condon et Ogden, 1991). Des centaines de jeunes ont accepté cette proposition et se sont installés en France métropolitaine. Les migrations féminines étaient composées dans la première période d'épouses, de compagnes ou de fiancées des hommes recrutés par les administrations; de plus en plus de femmes seules partaient également pour la métropole, par la filière des domestiques ou par les recrutements dans les hôpitaux ou d'autres établissements de soins (idem). L'action du Bumidom a élargi les possibilités de recrutement des femmes sur le marché de l'emploi métropolitain. Hommes et femmes étaient orientés, soit vers des centres de formation, soit vers des emplois dans le secteur privé, les administrations se chargeant de leurs propres recrutements de migrants des DOM par des concours d'entrée et par le placement en stages en métropole. Anselin (1979) a estimé que les migrations par le Bumidom ont compté pour plus du tiers des flux migratoires des DOM entre le milieu des années 1960 et les années 1970, nombre auquel il faut rajouter les installations en métropole de militaires du contingent démobilisés. La migration organisée a également suscité des centaines de départs spontanés, frères, sœurs, cousins, neveux, amis des femmes et hommes migrants placés par l'agence d'État.

18 Dans les sociétés caribéennes post-esclavagistes, la mobilité sociale a été acquise par l'accès à l'éducation et l'émigration (Patterson, 1978; Thomas-Hope, 1993). Si les migrations depuis 1945, notamment celles en direction des États-Unis (Thomas-Hope, 
1993) ont concerné les classes moyennes, la majorité des personnes ayant migré vers la France et la Grande-Bretagne est issue des classes populaires. La migration des membres de la famille constituait un investissement pour l'avenir. Comme pour l'ensemble des aspects de la vie quotidienne dans les Caraïbes, la famille faisait partie intégrante $\mathrm{du}$ processus migratoire à cette époque. Elle soutenait financièrement l'émigration, élevait ou donnait d'autres types d'aide aux personnes dépendantes qui restaient (Soto, 1987), et, en France et en Grande-Bretagne, la famille accueillait les migrants, les hébergeait et les aidait à s'adapter au nouvel environnement. Puis, tout particulièrement dans les îles anglophones, l'envoi d'argent par les migrants est devenu un élément majeur dans les revenus des pays. C'est justement une des nouvelles perspectives dans l'étude des migrations au cours de la période récente qui a révélé des modes de fonctionnement des familles (dont les familles caribéennes) souvent innovants suite à la dispersion géographique de leurs membres (Boyd, 1989 ; Bryceson et Vuorela, 2002 ; Chamberlain et Leydesdorff, 2004 ; Hareven, 2000 ; Olwig, 2001).

\section{Famille, résidence et logement dans les métropoles}

19 Les populations caribéennes installées dans les métropoles française et britannique au milieu des années 1960 se composaient alors de couples de migrants, femmes ou hommes venus seuls, dont certains étaient entrés en union après leur arrivée (avec un ou une compatriote ou avec une personne d'une autre origine), et de jeunes familles. Dans les deux cas, la résidence des migrants était principalement urbaine, localisée tout particulièrement dans les capitales ou les plus grandes villes. Les modes de recrutement ont largement influencé la répartition géographique des migrants (Peach, 1968 ; Condon et Ogden, 1993; Byron et Condon, 2008); par la suite, les réseaux familiaux ont contribué à accentuer les regroupements dans certaines régions. C'est en France métropolitaine que ces populations étaient le plus concentrées, $40 \%$ des femmes et $32 \%$ des hommes vivant en région parisienne en 1968 (33\% des femmes et $25 \%$ des hommes vivant dans Paris intra muros). De par leur affectation dans des bases militaires en province pendant le service militaire et par leur placement dans des emplois industriels, les hommes étaient moins localisés dans la capitale.

Le monde des migrants caribéens arrivés dans les deux métropoles à cette époque était celui du meublé, soit en hôtel, soit en chambre isolée. À Londres, Birmingham ou dans les autres régions d'arrivée en Grande-Bretagne, les hommes louaient là où se présentaient les opportunités près des gares ou selon des indications données par des compatriotes. Ceux qui étaient mariés ou avaient laissé une petite amie faisaient de leur mieux pour arranger le logement pour l'arrivée de leur femme ou fiancée. Ce fut souvent un choc, comme le laisse deviner la réaction de Hortense, personnage du roman d'Andrea Levy, qui rejoint Gilbert dans la chambre meublée à Londres : "C'est tout? $»^{5}$. À Paris, les déceptions devant les conditions de logement dans la «mère patrie » étaient similaires : Murielle rappelle sa réaction en découvrant le logement que son mari avait loué " grand comme une boîte d'allumettes; quand je pensais à ma chambre à la Martinique... ». D'après les témoignages de personnes arrivées des Antilles à cette époque, leur expérience a été marquée par des changements fréquents de chambre, l'absence de tout confort, l'interdiction de faire sa cuisine, de recevoir des amis. Même si les migrants avaient été prévenus des difficultés, il leur était impossible d'imaginer l'habitat dans les grandes villes françaises, l'exiguïté des logements, l'absence d'espace habitable extérieur ; comme le montre ce témoignage : 
"J'étais d'abord chez ma cousine, dans le 14 arrondissement (de Paris). Elle avait une petite chambre de bonne. C'était tellement dur pour faire la vaisselle, laver le linge, moi qui étais habituée à la grande terre, tout faire avec des grandes eaux au-dehors, et j'étais vraiment gênée, avec ce petit évier dans le coin de la chambre" (Henriette, arrivée de la Guadeloupe en 1962, âgée de 23 ans).

21 Les conditions de logement difficiles subies par les migrants caribéens qui arrivaient dans les deux métropoles dans les années 1950 n'étaient pas limitées à ces populations (Duclaud-Williams, 1978; Madge et Willmott, 1981). La majorité de ces migrants s'installaient dans les grandes villes, là où les besoins en main-d'œuvre étaient les plus nombreux. Le parc de logement dans les grands centres urbains souffrait d'un manque d'investissement privé et public pendant les années 1920-1930, malgré une demande de logements croissante et le contexte de la Seconde Guerre mondiale - en plus de la dévastation causée par les bombardements des villes britanniques - avait entraîné la dégradation des logements vieillissants et mal entretenus. Les ménages à revenus modestes ont particulièrement subi les effets de cette situation de crise et il a fallu attendre les grands programmes de construction de logements sociaux pour qu'un desserrement de la crise se produise. Toutefois, et surtout dans le cas britannique, les migrants n'étaient pas les premiers bénéficiaires. Pour pouvoir louer un logement plus confortable ou pour acheter, il fallait disposer de moyens financiers suffisants ; et pour obtenir un logement social, certaines conditions étaient imposées dont celle de la durée de résidence dans la commune. L'arrivée massive de migrants caribéens en GrandeBretagne, plus précoce qu'en France, s'est produite à une époque où la compétition sur le marché du logement bon marché était la plus forte. L'annonce de la nouvelle législation de 1962 a suscité l'augmentation de la proportion de personnes "dépendantes » (enfants, épouses...) parmi les migrants et le besoin de logements plus grands est devenu plus pressant. Nombreux sont les ménages qui ont été victimes de propriétaires peu scrupuleux qui profitaient de la crise du logement et de la naïveté des nouveaux arrivants pour exiger des loyers excessifs. Par ailleurs, les attitudes hostiles envers ces migrants ont été particulièrement vives et les attitudes racistes et discriminatoires dans la sphère du logement assez répandues, comme en témoignent de nombreuses études (Carter et al., 1987 ; Karn et al., 1986; Rex et Moore, 1967). Les premières années d'installation pour ces migrants ont donc été particulièrement difficiles et, en attendant un accès plus large au parc social, d'autres solutions ont été trouvées pour contourner les obstacles à la recherche d'un logement convenable (Byron et Condon, 2008). Frères, sœurs, cousins et autres proches ont souvent réuni leurs ressources afin d'acheter une maison délabrée dans les quartiers populaires centraux, la rénover et partager l'espace entre plusieurs ménages. Ce type de logement aurait souvent été un premier pas vers l'accès à un logement plus spacieux et mieux équipé en banlieue.

L'arrivée massive de migrants antillais en France métropolitaine, et notamment à Paris, a eu lieu plutôt dans les années soixante. Grâce à la construction de lotissements et des premiers grands ensembles, le départ vers la banlieue de centaines de ménages parisiens modestes avait déjà commencé. Entre temps, le parc des chambres meublées et des logements locatifs vétustes en centre-ville subsistait et était en grande partie occupé par des populations migrantes; mais le développement de bidonvilles en proche banlieue témoignait du volume insuffisant de logements à cette époque. Sort réservé aux familles algériennes ou portugaises, cette forme de logement n'a pas été connue par les ménages antillais. Dans son enquête menée en région parisienne à la fin des 
années soixante, Alain Anselin (1979: 179-206) a constaté que presque la moitié des ménages enquêtés à Paris vivait dans une seule pièce et que la grande majorité des petits logements habités par des Antillais était surpeuplée. Il a souligné le rôle des loyers élevés dans l'enfermement des Antillais dans le secteur des meublés ou des logements vétustes. Comme pour les immigrés étrangers, et pour les migrants caribéens à Londres ou Birmingham, les victimes des marchands de sommeil étaient nombreuses. Si les migrants antillais n'ont pas subi les mêmes formes de racisme dans leur recherche de logement que leurs homologues en Grande-Bretagne (Condon et Ogden, 1993), un observateur contemporain a noté: "C'est sans doute à travers la recherche d'un logement que l'Antillais prend le plus rapidement conscience de sa condition et des réalités de la vie métropolitaine " (Rudel, 1968). L'enquête d'Anselin a révélé que les ménages monoparentaux étaient particulièrement pénalisés. Ces ménages, la plupart du temps des femmes avec enfants, avaient souvent les revenus les plus bas et étaient retenus longtemps dans les îlots d'habitat vétuste des quartiers nord-est de Paris. Il est vrai aussi que ces femmes avaient souvent l'obligation d'envoyer de l'argent aux Antilles, soit pour arrondir le budget familial, soit pour financer les besoins d'un enfant resté chez les parents de la migrante.

Cette situation résidentielle a été celle d'une bonne partie de la population antillaise jusqu'à la fin des années soixante, très concentrée à Paris, en proche banlieue parisienne ou dans d'autres grandes villes. L'installation en banlieue s'est parfois faite d'entrée de jeu, notamment dans le cadre d'un hébergement par un membre de la famille ou l'attribution d'un logement pour certains employés des administrations mais elle a surtout été la conséquence du desserrement de la population parisienne vers l'habitat social de banlieue. Les immeubles appartenant aux grandes administrations et les logements réservés par le $1 \%$ patronal étaient accessibles aux employés des services publics, catégorie dans laquelle se situait une forte proportion de femmes et d'hommes antillais en métropole (Condon, 1995). Les ménages où les deux partenaires du couple travaillaient dans les services publics ou les entreprises nationalisées étaient particulièrement favorisés, non seulement parce qu'ils avaient tous deux des revenus stables mais aussi parce qu'ils avaient la possibilité de demander un logement social par l'un ou l'autre employeur. À l'époque, les demandes de logement étaient satisfaites assez rapidement, mais la localisation du logement attribué ne convenait pas toujours au demandeur, notamment lorsqu'il était éloigné du lieu de travail de l'un des conjoints ou localisé dans une commune mal desservie par les transports en commun. En général, le logement attribué était accepté au bout de deux ou trois propositions puisque l'essentiel, pour un ménage qui s'agrandissait, était de pouvoir bénéficier d'un logement plus spacieux et mieux équipé. Les autres filières, par la municipalité, par la préfecture ou par la caisse d'allocations familiales, avaient un impact limité pour les ménages antillais dans les années soixante, c'est-à-dire avant la libération d'un nombre croissant de logements sociaux avec les départs des ménages «métropolitains » des grands ensembles excentrés (Pinçon, 1981).

24 Dans les villes britanniques, les migrants caribéens qui souhaitaient accéder à un logement social n'ont pas bénéficié du même système d'accès par l'employeur. Par ailleurs, l'accès à ce secteur était beaucoup plus lent, à cause des résistances - et des discriminations raciales - s'opérant dans certaines municipalités. Comme pour la France, ce n'est que lorsque les ménages qui avaient pu s'y installer après la construction des immeubles ont commencé à quitter le parc social en masse que les ménages «immigrés» ont pu y accéder. Mais, de part et d'autre de la Manche, cette 
ouverture au milieu des années 1970 s'est accompagnée d'une tendance à la ségrégation résidentielle. Les grands ensembles de la région parisienne, de la inner city londonienne ${ }^{6}$ et près des zones industrielles de Birmingham ou d'autres villes ont enregistré un accroissement de la proportion de ménages à faibles revenus, de ceux où l'un des conjoints était chômeur de longue durée et enfin de ménages monoparentaux, catégories dans lesquelles les immigrés étrangers et les migrants caribéens étaient surreprésentés.

L'arrêt de l'immigration caribéenne en Grande-Bretagne a précédé l'accès de ces ménages au parc social. Malgré l'exiguïté des logements, les nouveaux migrants étaient hébergés par des proches parents ou des amis pendant quelques jours ou semaines en attendant de trouver une chambre. Dans le cas français, les arrivées se sont poursuivies jusqu'à aujourd'hui. Avec la durée de séjour, le nombre de membres de la parenté résidant dans les métropoles s'est accru; autant de personnes pouvant aider les nouveaux migrants à surmonter les obstacles du marché du logement. Les migrants antillais qui avaient eu accès à un logement plus grand, avec deux ou trois chambres, pouvaient les accueillir plus facilement. De 1960 à 1980, de nombreux ménages accueillaient en permanence les jeunes frères ou neveux démobilisés après le service militaire, les sœurs, cousines ou autres parents qui avaient migré pour trouver du travail, pour terminer leur scolarité en France métropolitaine ou pour suivre une formation. Les jeunes hébergés étaient souvent sollicités pour aider le ménage, notamment pour la garde de jeunes enfants. Le cas de George est exemplaire. Elle est partie de la Guadeloupe en 1957 pour rejoindre son mari qui travaillait depuis deux ans dans une usine fabriquant des automobiles à l'ouest de Paris. Les quatre premières années, ils ont habité dans diverses chambres meublées dans le $10^{\text {ème }}$ arrondissement de Paris et les deux premiers enfants sont nés pendant cette période. Ils ont trouvé un deux-pièces en 1961, puis George a "fait venir" sa jeune sœur pour s'occuper des enfants pour qu'elle puisse commencer à travailler comme agent hospitalier. En 1962, le couple a déposé une demande de logement social auprès des deux employeurs. À la naissance d'un quatrième enfant en 1965 , le couple a obtenu un logement social à Créteil (sud-est parisien). Juste avant, George avait commencé à travailler de nuit afin de partager la garde des enfants avec son mari. Entre temps, la sœur de George était partie s'installer ailleurs dans Paris. Par la suite, une jeune cousine est venue s'occuper des plus jeunes enfants tout en suivant des cours du soir. Ce cas illustre bien à la fois le surpeuplement des logements occupés par les ménages antillais pendant les premières années du séjour, les stratégies employées pour que les deux partenaires du couple puissent travailler à plein temps et fonder une famille, et le rôle des réseaux familiaux dans les courants migratoires. Ces expériences sont communes à d'autres migrations. Cependant, ce rôle d'accueil des nouveaux venus n'est pas sans générer des tensions au sein des ménages.

Le logement social a été particulièrement recherché comme solution résidentielle par les ménages caribéens dans les deux contextes nationaux (Annexe 2). S'ils gagnaient en terme d'espace et de confort, ils perdaient très souvent en terme de localisation par rapport au lieu de travail. En cas de perte d'emploi, résider dans une banlieue excentrée pouvait rendre la recherche d'un nouvel emploi plus difficile et plus coûteuse. Jusqu'au début des années 1990, les populations antillaises en France métropolitaine, largement employées dans la fonction publique, étaient beaucoup moins touchées que leurs homologues en Grande-Bretagne (Byron et Condon, 2008). La désindustrialisation à partir de la fin des années 1970 et la privatisation des services publics britanniques des 
années 1980 a notamment affecté l'emploi des hommes caribéens dans ce pays. En attendant de trouver un nouvel emploi ou une formation, les hommes en couple dont la conjointe avait un emploi se sont trouvés certainement dans une situation moins précaire que ceux qui vivaient seuls. En même temps, les femmes qui travaillaient dans des secteurs en voie de privatisation, par exemple dans la restauration auprès des services publics, se sont trouvées dans des situations d'emploi instables et le temps partiel subi est devenu plus fréquent. Pour celles qui assurent seules la charge d'une famille, le soutien d'un réseau familial ou de voisinage est devenu de plus en plus nécessaire.

\section{Dynamiques des ménages et des familles dans des sociétés en pleine transformation sociale et dÉmographique}

\section{Mariages et familles nucléaires en contexte migratoire}

Pendant la première décennie des migrations étudiées ici, le processus des chaînes migratoires a entraîné le regroupement de membres de la famille étendue au sein des ménages. Le contexte du logement a participé à ces regroupements. La composition des populations migrantes, de jeunes femmes et hommes vivant très souvent en couple, était le reflet à la fois de la structure démographique de ces groupes et de la pression des normes sociales. Certains de ces couples étaient déjà constitués au moment de l'émigration: les conjoints avaient migré ensemble ou s'étaient retrouvés après quelques mois ou années de séparation; d'autres étaient composés de personnes qui s'étaient rencontrées dans les métropoles, parfois des compatriotes, sinon des personnes d'autres origines. Ce qui est frappant, c'est la forte proportion de couples mariés.

Avant leur départ, ces migrants avaient intériorisé un nombre de représentations contrastées sur la famille. D'un côté, les représentations occidentales de la sexualité des populations caribéennes et de l'instabilité des couples; de l'autre, les formes du couple et de la famille symboles de la respectabilité bourgeoise et de la moralité victorienne (dans le cas britannique) ou catholique/chrétienne, sinon de la tradition républicaine (Charbit, 1987). Le lien entre mariage et mobilité sociale était une évidence pour grand nombre de migrants. En arrivant dans les villes britanniques ou françaises, ils découvraient que le mariage était également la norme parmi les classes ouvrières. Ainsi, les unions consensuelles devenaient acceptables seulement si elles étaient formalisées par le mariage. Le témoignage de cette femme de l'île de Nevis illustre bien la pression sociale : «I Ici, ça se faisait, on se mariait. Même les filles qui n'étaient pas mariées, quand il fallait aller chercher les enfants à l'école, on mettait une alliance. Toutes les mères voulaient donner l'impression qu'elles étaient mariées " (Byron et Condon, 2008).

Si les enquêtes du PSI ont révélé que les migrants caribéens étaient moins souvent mariés que les autres résidents de Grande-Bretagne (Brown, 1984), leurs taux de mariage étaient plus élevés que dans les mêmes cohortes d'âge dans les îles caribéennes. Cette première génération de migrants avait largement opté pour le mariage : au recensement de 1991, dans le groupe âgé de 60-64 ans, $56 \%$ des femmes et $68 \%$ des hommes étaient mariés et $31 \%$ des femmes et $19 \%$ des hommes étaient divorcés ou veufs. En France métropolitaine aussi, on observe une tendance similaire. 
Au recensement de 1968 , près de la moitié des hommes $(46,7 \%)$ et $41 \%$ des femmes étaient mariés et $5 \%$ veufs. Malheureusement, la publication des données du recensement de 1968 ne permet pas de restituer la répartition de la population antillaise par type de ménage. Nous savons toutefois qu'environ $60 \%$ des ménages étaient composés de parents et d'enfants et le tiers de ces ménages, de trois enfants ou plus. Au cours des quinze années suivantes, la taille des familles s'est réduite, reflétant aussi l'évolution de la fécondité aux Antilles (Charbit, 1987b).

Malgré des conditions de logement difficiles, la plupart des jeunes ou nouveaux couples ont pris la décision de fonder une famille. Les témoignages de cette période soulignent l'isolement ressenti par les hommes et les femmes par rapport à l'environnement familial qu'ils avaient connu dans les Caraïbes. On évoque l'aide précieuse des parents ou d'autres ascendants au moment des premières naissances, surtout pour celles survenues en dehors d'une union stable. Le cas suivant est très éclairant : " Ici, on était tout seuls. Quand nous avons eu Jasmin, notre troisième, Robert et moi, on ne savait pas comment faire. On a dû se débrouiller. Imagine, je me sentais perdue et j'avais déjà deux enfants. Mais quand j'habitais avec ma mère, chez nous, c'est elle qui s'occupait de tout. Pour la deuxième enfant, c'est les parents de Robert qui l'ont prise quand elle avait un an ». À travers les témoignages de l'organisation familiale, le rôle central des femmes dans le soutien aux divers membres de la famille est évident, même lorsque leurs filles, en tant que mères, deviennent « adultes ». Dans les familles de la première génération de migrants, il était courant que la sœur aînée se substitue à la mère lorsqu'il y avait beaucoup d'enfants dans la famille ou lorsque la mère était très malade. Puis, avec l'allongement de la vie, l'aide aux parents vieillissants émerge comme rôle joué en même temps que celui du soutien de la jeune génération (Attias-Donfut et Lapierre, 1998).

31 L'expérience de la migration et de la nouvelle structure familiale a semblé induire de profonds changements au niveau des rapports de genre et des rôles assumés dans la famille. À la différence du contexte caribéen, les tâches domestiques étaient souvent partagées - au moins dans les premiers temps - par les deux membres du couple. Dans certains secteurs de l'emploi, les migrants avaient la possibilité de travailler la nuit ou de commencer très tôt le matin. C'était notamment le cas dans le secteur hospitalier, dans les transports ou à la poste, et aussi dans le travail en usine. Mari et femme pouvaient s'organiser alors pour se relayer, assurant ainsi la garde et l'éducation des enfants (Byron et Condon, 2008). Les forts taux d'activité féminine dans ces populations (Byron, 1998; Condon, 2004), en plus d'un désir d'autonomie chez les femmes, reflétaient les stratégies des couples de créer un foyer confortable et un lieu pour élever leurs enfants et en même temps de maintenir les liens avec le pays d'origine et d'envoyer de l'argent à la famille restée.

32 En parallèle, les normes liées aux rôles dans la famille évoluaient dans la société en France et en Grande-Bretagne : les femmes exigeaient plus d'autonomie et de contrôle sur leur corps et leur vie quotidienne. Ces désirs allaient influer sur les formes du ménage et de la famille. La «famille nucléaire » commençait à perdre sa place en tant qu'unité principale de la parenté en Europe et en Amérique du Nord. La transformation des attitudes et des valeurs socioculturelles expliquerait un ensemble de changements démographiques : la réduction de la taille des ménages, l'augmentation du nombre de personnes qui choisissent de vivre seules, le report du mariage ou de la mise en couple, la croissance de la divortialité, la hausse de l'âge au premier enfant, la baisse de la fécondité, l'institution de couples de personnes du même sexe (Ogden et Hall, 2004). Si 
certains y voient les signes d'une déstabilisation des structures familiales ("traditionnelles»), d'autres observent plutôt les nouvelles formes de parenté et de réseaux d'amitié (Leridon et Villeneuve-Gokalp, 1994 ; Villeneuve-Gokalp, 1997). L'expérience familiale des originaires des Caraïbes vivant dans les deux sociétés étudiées ici doit être analysée dans ce contexte en pleine évolution, tout en tenant compte du rôle de la classe sociale, de la génération et des rapports de genre et en examinant les tendances démographiques dans les îles caribéennes.

\section{Séparation et divorce}

33 L'une des conséquences de l'adoption de la norme du mariage dans la première génération de migrants caribéens semble être l'émergence dans les statistiques du recensement d'un nombre élevé de divorces. En plus des facteurs qui conduisent à la séparation des couples qui peuvent prendre de l'importance en situation migratoire - par exemple les tensions liées aux conditions de logement, aux désaccords sur les projets du couple, ou à l'infidélité (Byron et Condon, 2008) -, l'enregistrement civil d'une séparation par un divorce est lié à la formalisation de l'union par le mariage. L'observation de taux de divorce significativement plus élevés dans les populations caribéennes que dans l'ensemble de la population adulte britannique, et notamment parmi les plus âgés, a conduit Berthoud et Beishon (1997) à conclure que la croissance du nombre des mariages qui se terminent par un divorce s'est manifestée plus tôt que dans la population "blanche ». Cependant, une comparaison par génération n'indique pas d'augmentation des taux parmi les personnes d'origine caribéenne, puisque les plus jeunes, contrairement aux Britanniques du groupe majoritaire, sont moins souvent divorcés que leurs aînés (Annexe 3a). Pour la génération plus ancienne, ces séparations s'inscriraient dans la continuité des comportements de couple de leur génération, et de celle d'avant, au moment de leur émigration ; car si la séparation définitive d'un couple marié se traduit par un divorce, celle de couples vivant en union libre ne laisse pas de trace dans l'état civil.

Un résultat très intéressant de la comparaison internationale est la similitude des tendances. Comme pour les Caribéens en Grande-Bretagne, l'analyse des données pour la population antillaise vivant en France métropolitaine révèle une augmentation du nombre de divorcés entre les années soixante et $1990:$ de $1,5 \%$ des hommes âgés de 15 ans ou plus en 1968 à 4,9\%, et une hausse pour les femmes de 2,3\% à 7,5\%. Et plutôt qu'un accroissement de la tendance au divorce, cette évolution traduit la forte divortialité dans la génération des premiers migrants de travail : en 1990, 15,1\% des femmes antillaises âgées de 60-64 ans étaient divorcées contre 10,3\% parmi les 35-39 ans ; et les proportions pour les deux groupes d'âges chez les hommes étaient de $11,1 \%$ et $6,3 \%$. Bien évidemment, certains couples plus jeunes divorceront entre 40 et 60 ans ; cependant, la comparaison avec les jeunes générations suggère une nouvelle tendance dans l'ensemble de la population métropolitaine, vers moins de mariages et donc moins de divorces. L'écart entre les taux pour la population antillaise et l'ensemble de la population en France métropolitaine est très marqué pour cette génération plus âgée : la proportion de divorcés est plus de deux fois plus élevée parmi les natifs des Antilles, tandis que dans la génération plus jeune, les taux sont très proches (Annexe $3 b$ ). La baisse de nuptialité parmi les populations antillaises se traduit par moins de personnes mariées dans le groupe d'âge 35-39 ans et plus de personnes célibataires, tendance qui se retrouve, à un moindre degré encore, dans l'ensemble de 
la population en France. En Grande-Bretagne aussi, la proportion de célibataires dans la plus jeune génération est plus élevée pour la population classée comme "blanche", mais elle est surtout élevée dans le groupe d'origine caribéenne (Annexes 3a et 3b).

En dehors des enquêtes examinant ce processus, le remariage des personnes divorcées n'est pas visible dans les répartitions de la population par état civil. Cette invisibilité statistique touche plus la population masculine, car ce sont les hommes qui ont eu tendance à se remarier le plus. Mais ce n'est pas toujours le cas et l'isolement ressenti par les hommes vivant seuls, qui s'aggrave avec l'âge, est un phénomène qui touche de nombreux hommes d'origine caribéenne (Plaza, 2001). Les femmes, étant plus intégrées dans des réseaux sociaux et plus impliquées dans les affaires de la famille élargie, sont beaucoup moins isolées même après un divorce. Et ce sont elles qui sont en général au cœur des relations familiales, avec lesquelles les enfants ont le plus de contacts et qui jouent un rôle important dans la garde des petits-enfants (Byron et Condon, 2008). Si de nombreux parents se mettent en couple avec un nouveau partenaire, la séparation des couples ayant des enfants entraîne la constitution de nouveaux ménages monoparentaux. Ces séparations peuvent avoir lieu à divers âges des parents, à divers moments du cycle de vie de la famille. La représentation des «familles monoparentales » caribéennes est celle d'une jeune mère qui a vécu peu de temps avec le père de son ou de ses enfants. Dans les métropoles européennes, ce n'est qu'un des cas de figure des «familles caribéennes » mais c'est celui qui domine encore parmi les représentations de ces populations. L'analyse de ces tendances nous amène à nous intéresser aux formes familiales des migrants caribéens et de leurs descendants en Europe aujourd'hui.

\section{Les familles caribéennes « monoparentales » en Europe}

Les analyses précédentes indiquent un changement de comportement intergénérationnel. Si les migrants des années 1950 et 1960 avaient adopté le modèle du mariage, leurs descendants semblent être en train de recréer les formes familiales plus courantes dans les îles caribéennes. En Grande-Bretagne, les faibles taux de mariage dans la population originaire des Caraïbes s'accompagnent d'un développement croissant des ménages monoparentaux. Ceux-ci constituaient en 2001 près du quart des ménages dont le responsable était enregistré comme "caribéen" contre $44 \%$ des ménages composés d'une famille - un taux trois fois plus important que dans la population catégorisée comme «blanche». En France métropolitaine aussi, la proportion de ces ménages s'accroît depuis les années 1970, de 11,5\% de l'ensemble des ménages en 1975, à 13,4 \% en 1982 et à 16,2 \% des ménages en 1999. Une augmentation similaire est observée dans la catégorie des employées, comme Charbit l'avait déjà signalé (1987b) : en 1982, 13,2\% des ménages dont la personne de référence était classée comme employée étaient des ménages monoparentaux dont la responsable était une femme et en 1999, la proportion s'élevait à $22 \%$. Ainsi, la comparaison entre les ménages «antillais » et l'ensemble des ménages révèle une proportion deux fois plus importante de ménages monoparentaux parmi les Antillais (respectivement 16,2\% et $6,8 \%$ mais l'examen des distributions selon le groupe social indique que des dynamiques autres que "culturelles » jouent un rôle dans la construction des normes familiales : les ménages "antillais», dont une bonne partie est classée dans le groupe des « employés », se rapprocheraient du comportement de leur groupe social en France métropolitaine. 

quartier; la concentration géographique des ménages « antillais » et la résidence dans les mêmes grands ensembles, conséquence des filières d'attribution des HLM par l'employeur dans les années 1960-70 puis par la préfecture et la CAF dans les années 1970-80. Tout de même, s'ils sont sur-représentés parmi les ménages "antillais ", les ménages monoparentaux sont loin d'être la forme majoritaire. Malgré ceci, les bailleurs sociaux en France, ayant parmi leurs locataires une proportion importante de ces ménages, ont commencé à repousser ces «familles monoparentales " considérées comme des «familles à problèmes ». En effet, dans les deux pays, ces familles ont souvent été victimes de pratiques discriminatoires en termes d'attribution de logements, obtenant les appartements les moins bien situés ou de moins bonne qualité (Peach et Byron, 1993 ; Condon, 1995). Si le ménage monoparental est une réalité pour de plus en plus de femmes et d'enfants, le vrai contenu ou les contours de cette situation familiale sont rarement examinés en profondeur (Reynolds, 2005). La famille étendue et les réseaux sociaux sont notamment ignorés dans des analyses qui se focalisent sur le degré de contact avec le père des enfants ou sur la marginalisation des mères et des enfants. En parallèle, les agences de l'État continuent à chercher des explications à ce "phénomène socioculturel»-dont les grossesses parmi les adolescentes et le «comportement irresponsable» des jeunes hommes seraient des dimensions notables - dans le but de réaffirmer la norme des ménages bi-parentaux. Un aspect qui est souvent négligé est le choix de vivre seul-e avec ses enfants : pour de plus en plus de femmes, le désir de plus d'autonomie les pousse à rejeter la vie de couple sans pour autant rejeter l'idée de devenir mère.

\section{Changements et continuités dans l'histoire de la famille caribéenne}

Cette analyse de la famille à travers l'histoire de deux migrations parallèles a voulu mettre en évidence la manière dont des contextes particuliers peuvent façonner les formes familiales. La migration est un phénomène qui, non seulement est généré et perpétué par des dynamiques familiales mais aussi entraîne des changements profonds ou temporaires. Une dimension particulière ici est le contexte (post)colonial, qui a suscité les flux migratoires et orienté les devenirs professionnels et résidentiels des migrants, et également influencé le regard de la société coloniale sur les formes familiales des descendants d'esclaves caribéens. L'instabilité du couple caribéen continue à intriguer chercheurs et politiques et est souvent montrée du doigt dans l'explication de la pauvreté des ménages monoparentaux ou de la moindre réussite scolaire des jeunes hommes descendants de migrants caribéens. Une telle perspective ne tient pas compte des solidarités familiales qui existent au-delà du ménage et, en focalisant l'attention sur un seul aspect de la vie des familles, occulte d'autres facteurs d'exclusion.

La comparaison internationale est d'un grand apport à l'analyse et à la compréhension des transformations étudiées ici. En la comparant à une migration de travail fort bien documentée, elle resitue la migration antillaise vers la France métropolitaine dans son contexte régional, historique, sociopolitique et culturel. Les émigrants caribéens ont quitté une région marquée par une histoire coloniale proche. Les liens particuliers ont orienté les flux vers l'une ou l'autre métropole "mère patrie ». Les individus et les

Revue européenne des migrations internationales, vol. 24 - $n^{\circ} 1$ | 2008 
couples se sont adaptés à un contexte social nouveau : milieu urbain, logements exigus, éloignement des membres de la famille élargie, norme du mariage dans la classe ouvrière. Cette première génération, avec ses espoirs de promotion sociale, a du faire des sacrifices et a été confrontée à de multiples obstacles mais s'est efforcée de donner un avenir meilleur à ses enfants. La génération des descendants est arrivée à l'âge adulte dans un tout autre contexte. Les jeunes femmes et hommes ont bénéficié d'une scolarisation plus longue, envisagent rarement de faire les mêmes métiers que leurs parents mais se retrouvent face à un marché de l'emploi plus tendu. Par ailleurs, les rapports entre hommes et femmes ont beaucoup évolué depuis la jeunesse de leurs parents, les femmes ayant obtenu de nouveaux droits, notamment sur leur corps. Les calendriers de la mise en couple et de la venue du premier enfant se diversifient; les femmes choisissent de plus en plus de vivre seules ; et ces bouleversements sociaux se ressentent dans tous les milieux. Paradoxalement, les formes familiales des Caribéens en Grande-Bretagne et en France tendent à se rapprocher du système familial prévalant dans la région caribéenne dans les années cinquante : échanges au quotidien entre les générations (notamment entre les femmes), de nombreuses femmes élevant seules leurs enfants, mariages peu importants. Dans le contexte européen, les membres des familles « caribéennes » élargies sont parfois éloignés géographiquement dans les villes mais fonctionnent comme soutien aux jeunes (étudiants, petits-enfants, chômeurs, parents seuls) et, de plus en plus, aux aînés. Ce sont ces dimensions de la «famille caribéenne » qu'il faut analyser en profondeur.

Aujourd'hui, les communautés caribéennes en Grande-Bretagne et en France métropolitaine s'étendent au-delà de la population migrante et de ses descendants. Certains migrants se sont mis en couple avec des personnes d'origine métropolitaine et ces couples sont de plus en plus fréquents ${ }^{7}$. Ainsi, les «familles caribéennes » en Europe intègrent très souvent des personnes n'ayant aucun lien avec les Caraïbes, fait qui souligne l'importance de la dimension sociale - et pas seulement culturelle - dans les formes familiales.

41 Cette histoire des migrations est aussi une histoire de familles. Des familles qui, à travers des solidarités diverses parviennent à surmonter de nombreux obstacles et qui fonctionnent en tant que "familles transatlantiques". Plutôt que des familles " désorganisées », il s'agit davantage de familles qui s'organisent et se réorganisent en permanence.

\section{BIBLIOGRAPHIE}

ANSELIN Alain (1979) L'émigration antillaise en France, Paris, Anthropos.

ATTIAS-DONFUT Claudine et LAPIERRE Nicole (1998) La famille providence. Trois générations en Guadeloupe, Paris, La Documentation Française (avec la CNAV).

BARROW C (1996) Family in the Caribbean: themes and perspectives, Ian Randle, Jamaica and James Currey, Oxford. 
BERTHOUD R. et BEISHON S. (1997) People, families and households, in Modood, T, Berthoud, R. et al, Ethnic Minorities in Britain: diversity and disadvantage, PSI, London, pp. 19-59.

BOUGEROL Christiane (1998) Les hommes en prison à la Guadeloupe, Ethnologie française. BOYD Monica (1989) Family and personal networks in international migration: recent developments and new agendas, International Migration Review, 23, pp. 638-670.

BROCK C., Dir. (1986) The Caribbean in Europe. Aspects of the West Indian experience in Britain, France and the Netherlands, London, Editions Frank Cass.

BRYCESON Deborah and VUORELA Ulla, Eds. (2002) The transnational family, Berg, Oxford.

BURNARD Trevor (2006) "Rioting in goatish embraces": marriage and improvement in early British Jamaica, The History of the Family, 11 (4), pp. 185-197.

BYRON Margaret (1998) Migration, Work and Gender: the case of post-war labour migration from the Caribbean to Britain, in Chamberlain, M. (Ed.) Caribbean Migration: Global Identies, London, Routledge, pp. 217-231.

BYRON Margaret and CONDON Stéphanie (1996) A comparative study of Caribbean return migration from Britain and France: towards a context dependent explanation, Transactions of the Institute of British Geographers, 21, pp. 91-104.

BYRON Margaret and CONDON Stéphanie (2008) Migration in comparative perspective. Caribbean communities in Britain and France, London, New York, Routledge, 288 p.

CARNEGIE Charles (1987) A social psychology of Caribbean migrations: strategic flexibility in the West Indies, in Levine B B (dir.), The Caribbean exodus, Praeger, NY.

CARTER B., HARRIS C. and JOSHI S. (1987) The 1951-1955 Conservative government and the racialisation of black immigration, Policy Papers in Ethnic Relations n¹1, University of Warwick, Centre for Research in Ethnic Relations.

CHAMBERLAIN Marie et LEYDESDORFF Selma (2004) Transnational families : memories and narratives, Global networks, 4(3), pp. 227-241.

CHARBIT Yves (1987), Famille et nuptialité dans la Caraibe, Paris, PUF/INED, Travaux et Documents, Cahier 119.

CHARBIT Yves (1987b), Ménages et familles des originaires des départements d'outre-mer, Revue Européenne des Migrations Internationales, 3(3), pp. 49-67.

CHARBIT Yves et LERIDON Henri (1980) Transition démographique et modernisation en Guadeloupe et en Martinique, Paris, PUF/INED, Travaux et Documents, Cahier 89.

CGP (Commission Générale du Plan) (1959) Troisième Plan, 1958-1961. Rapport général de la Commission de la Modernisation et d'Équipement des Départements d'Outre-Mer, Paris, Imprimerie Nationale.

CLARKE Edith (1957) My mother who fathered me, George Allen and Unwin, London (réédition, 1970).

CONDON Stéphanie (2004) Gender issues in the study of circulation between the Caribbean and the French metropole, Caribbean Studies, 32 (1), pp. 129-159.

CONDON Stéphanie (1995) L'accès au logement : filières et blocages. Le cas des Antillais en France et en Grande-Bretagne, Rapport au Ministère de l'Équipement, Plan Construction et Architecture, Collection Recherches. 
CONDON Stéphanie and OGDEN Philip (1991) Emigration from the French Caribbean: the origins of an organised migration, International Journal of Urban and Regional Research, 15(4) pp. 505-523.

CONDON Stéphanie and OGDEN Philip (1993) The state, housing policy and Afro-Caribbean migration to France, Ethnic and Racial Studies, 16, pp. 256-297.

CONSTANT Fred (1987) La politique française de l'immigration antillaise de 1946 à 1987, Revue Européenne des Migrations Internationales, 3(3) pp. 9-29.

DAGENAIS H. et POIRIER J. (1985) L'envers du mythe : la situation des femmes en Guadeloupe, Nouvelles Questions Féministes, 9-10, pp. 53-83.

DAVISON Robert.B. (1966) Black British: immigrants to England, Oxford University Press, London. DOMENACH Hervé (2002) L'évolution au XXe siècle du système démographique et migratoire caribéen, Hommes et Migrations, 1237 ( $n^{\circ}$ spécial Diasporas caribéennes), pp. 13-25.

DOMENACH Hervé et PICOUET Michel (1992) La dimension migratoire des Antilles, Paris, Economica. DUCLAUD-WILLIAMS R.H. (1978) The politics of housing in Britain and France, London, Heinemann.

DUNCAN S. and EDWARDS, Eds. (1997) Single mothers in an international context: mothers or workers? London, UCL Press.

ELLIS Pat Ed. (1986) Women of the Caribbean, London et New Jersey, Zed Books Ltd.

FRAZIER E.F. (1940) The Negro family in the United States, Chicago, Chicago University Sociological Series.

FRYER Peter (1984) Staying Power: the History of Black People in Britain, London, Pluto Press.

GAUTIER Arlette (1993) Femmes seules et prestations sociales en Guadeloupe, in A. Gautier et J. Heinen (éds.) Le sexe des politiques sociales, Paris, Éditions Côté Femmes.

GAUTIER Arlette (2004) Procréation et famille en Guadeloupe et dans la Caraïbe, Espace, Populations, Sociétés, 2, pp. 207-219.

GIRAUD Michel (1997) Entre particularités épidémiologiques et spécificités culturelles : l'enquête sur les comportements sexuels aux Antilles et en Guyane française (ACSAG), Sciences Sociales et Santé, 15 (4), pp. 77-93.

GOULBOURNE Harry (1999) The transnational nature of Caribbean kinship in Britain, in S. McRae Ed. Changing Britain, Oxford, Oxford University Press.

GOULBOURNE Harry et CHAMBERLAIN Mary (2001) Caribbean families in Britain and the Transatlantic World, London, Macmillan.

HAREVEN Tamara (2000) Families, history and social change: life course and cross-cultural perspectives, Boulder, Colorado, Westview Press.

HILL COLLINS Patricia (1994) The tie that binds: race, gender, US violence, Ethnic and Racial Studies, 21(5), pp. 917-938.

INSEE (1970) Recensement de 1968. Population née dans un département ou territoire d'outre-mer et résidant en métropole, Paris, INSEE.

LEFAUCHEUR Nadine (1987) Les familles monoparentales : une catégorie spécifique? Paris, Plan Construction.

LEFEVRE Cécile et FILHON Alexandra Éds. (2005) Histoires de familles, histories familiales. Les résultats de l'enquête Famille de 1999, Les Cahiers de l'Ined, 156, Paris, Ined. 
LERIDON Henri et VILLENEUVE-GOKALP Catherine (1994) Constance et inconstances de la famille: biographies familiales des couples et des enfants, Paris, PUF/INED, Travaux et Documents Cahier 134.

LEVY Andrea (2004) A small island, London, Review.

MACLEOD C. and DURRHEIM K. (2002) Racializing teenage pregnancy: "culture" and "tradition" in the South African scientific literature, Ethnic and Racial Studies, 25 (5), pp. 778-801.

MADGE C. and WILLMOTT P. (1981) Inner city poverty in Paris and London, London, Routledge and Kegan Paul.

MARIE Claude-Valentin (1993) Populations nées et originaires des DOM-TOM, nées et originaires, résidant en France métropolitaine (résultats du sondage au quart), INSEE, Série Démographie et Société.

MARIE Claude-Valentin et RALLU Jean-Louis (2004) Migrations croisées entre DOMs et métropole : l'emploi comme moteur de la migration, Espace, Populations et Sociétés, 2, pp. 237-252.

MEULDERS-KLEIN Marie Thérèse et THERY Irène Éds. (1993) Les recompositions familiales aujourd'hui, Paris, Nathan (Essais et Recherches).

MILES Robert (1984) The riots of 1958: the ideological construction of 'race relations' as a political issue in Britain, Immigrants and Minorities, 3(3), pp. 252-275.

MILIA Monique (2002) De l'outre-mer au continent : étude comparée de l'émigration portoricaine et antillo-guyanaise de l'après-guerre aux années 1960, Thèse de troisième cycle, Paris, École des Hautes Études en Sciences Sociales.

MODEL S. and FISHER G. (2002) Unions between blacks and whites : England and the US compared, Ethnic and Racial Studies, 25(2), pp. 728-754.

MULOT Stéphanie (2000) «Je suis la mère, je suis le père » : l'énigme matrifocale. Relations familiales et rapports de sexes en Guadeloupe, Thèse de troisième cycle, Paris, École des Hautes Études en Sciences Sociales.

MURCH A. (1971) Black Frenchmen: the political integration of the French Antilles, Cambridge, Massachussets, Schenkman.

OGDEN Philip. E. and HALL R. (2004) The second demographic transition, new household forms and the urban population of France during the 1990s, Transactions, Institute of British Geographers, New Series, 29 (1), 88-105

OLWIG K. F. (2001) New York as a locality in a global family network, in Foner, N. Islands in the City, University of California Press, Berkeley, pp. 142-160.

OWEN D. (2001) A profile of Caribbean households and families in Great Britain, in Goulbourne H. and Chamberlain M., Caribbean families in Britain and the Transatlantic World, London, Macmillan, pp. 65-91.

PATTERSON Orlando (1978) Migration in Caribbean societies: a socio-economic and symbolic resource, in McNeill WH et Adams RS (dir.), Human migration, patterns and policies, Bloomington and London, Indiana University Press.

PEACH Ceri (1968) West Indian Migration to Britain: a social geography, London, OUP for the Institute of Race Relations.

PEACH Ceri (1991) The Caribbean in Europe: contrasting patterns of migration and settlement in Britain, France and the Netherlands, Research Paper in Ethnic Relations $n^{\circ} 15$, University of Warwick, Centre for Research in Ethnic Relations. 
PEACH Ceri et BYRON M. (1993) Caribbean tenants in council housing: "race", class and gender, New Community, 19 (3), pp. 407-423.

PHOENIX A. (1993) The social construction of teenage motherhood: a black and white issue?, in A. Lawson and D. L. Rhode (dirs.) The politics of pregnancy: adolescent sexuality and public policy, New Haven and London, Yale University Press, pp. 74-97.

PHOENIX A. et WOOLLETT A. (1991) Motherhood: social construction, politics and psychology, in A. Phoenix, A. Woollett et E. Lloyd dirs. Motherhood: meanings, practices and ideologies, London, Sage, pp. 13-45.

PINÇON Monique (1981) Les immigrés et les HLM. Le rôle du secteur HLM dans le logement des immigrés en Ile-de-France - 1975, Paris, Centre de Sociologie Urbaine (CNRS).

PLAZA Dwaine (2001) Ageing in Babylon: elderly Caribbeans in Britain, in Goulbourne H. and Chamberlain M. Caribbean families in Britain and the Transatlantic World, Macmillan, London, pp. 219-231.

POURETTE Dolorès (2006) Des Guadeloupéens en Ile-de-France. Identité, sexualité, santé, Paris, Karthala, coll. Médecines du Monde.

PRYCE K. (1979) Endless pressure. A study of West Indian lifestyles in Bristol, Bristol, Bristol Classical Press.

REDDOCK R. et al. (1999) Women and family in the Caribbean: historical and contemporary considerations, Caricom, Guyana.

REYNOLDS T. (2005) Caribbean mothers: identity and experience in the UK, London, Tufnell Press.

REX John and MOORE Robert (1967) Race, community and conflict, London, Oxford University Press for the Institute of Race Relations.

ROBERTS G.W. (1975) Fertility and mating in four West Indian populations, Jamaica, Institute of Social and Economic Research, University of the West Indies.

RUDEL Christian (1968) Un monde méconnu : les Antillais de métropole, Hommes et Migrations Documents, 736, pp. 1-10.

SENIOR Olive (1991) Working miracles: womens' lives in the English speaking Caribbean, London, James currey.

SIMEY T.S. (1946) Welfare and Planning in the West Indies, Oxford University Press, London.

SMITH Raymond T. (1988) Kinship and class in the West Indies: a genealogical study of Jamaica and Guyana, Cambridge University Press, Cambridge.

SMITH Raymond T. (1996) The Matrifocal family, in Smith, R. T. The Matrifocal Family: Power, Pluralism and Politics, London, Routledge, pp. 39-57.

SMITH Raymond T. (2001) Caribbean families: questions for research and implications for policy, in Goulbourne H. and Chamberlain M., Caribbean families in Britain and the Transatlantic World, London, Macmillan, pp. 48-62.

SOLIEN N.L. (1960) Household and family in the Caribbean, Social and Economic Studies, 9 (1), pp. 101-106.

SOTO I.M. (1987) West Indian child fostering: its role in migrant exchanges, in Sutton, C. and Chaney, E. M. (dir.), Caribbean life in New York City: socio-cultural dimensions, New York, Centre for Migration Studies, pp. 131-149. 
THOMAS-HOPE Elisabeth (1986) Transients and settlers: varieties of Caribbean migrants and the socio-economic implications of their return, International Migration, 24, pp. 559-571.

THOMAS-HOPE Elisabeth (1993) Explanations in Caribbean migration, London, Macmillan.

THOMPSON Paul et BAUER Elaine (2005) Sources of aid and resilience and points of pain in Jamaican migrant families, Revue Européenne des Migrations Internationales, 21(3), pp. 9-26.

VERTOVEC Steven (2003) Migration and other modes of transnationalism: towards conceptual cross-fertilization, International Migration Review, 37 (3), pp. 641-665.

WILSON P.J. (1973) Crab antics. The social anthropology of English-speaking Negro societies of the Caribbean, Yale University Press.

\section{ANNEXES}

\section{Annexe 1 : Effectifs de population née dans les îles caribéennes et installée dans les métropoles selon le sexe}

1a. Angleterre et Pays de Galles, recensements de 1951-2001

\begin{tabular}{|l|l|l|l|}
\hline Année & Femmes & Hommes & Ensemble \\
\hline 1951 & 6290 & 9011 & 15301 \\
\hline 1961 & 75992 & 95804 & 171796 \\
\hline 1971 & 151825 & 152245 & 304070 \\
\hline 1981 & 150755 & 144424 & 295179 \\
\hline 1991 & 139010 & 125581 & 264591 \\
\hline 2001 & 137637 & 115539 & 253176 \\
\hline
\end{tabular}

Sources: OPCS Census of Great Britain 1951-1991, Country of Birth Tables; ONS, Census 2001, Ethnicity and Country of Birth Tables.

1b. Population née aux Antilles et résidant en France métropolitaine aux recensements de 1954 à 1999

\begin{tabular}{|l|l|l|l|}
\hline & \multicolumn{3}{|l|}{ Nés à la Guadeloupe ou à la Martinique } \\
\hline \hline Année & Femmes & Hommes & Ensemble \\
\hline 1954 & $*$ & $*$ & 15620 \\
\hline
\end{tabular}




\begin{tabular}{|l|l|l|l|}
\hline 1962 & 16000 & 22000 & $37591^{* *}$ \\
\hline 1968 & 28556 & 32604 & 61160 \\
\hline 1975 & 57185 & 58280 & 115465 \\
\hline 1982 & 93256 & 89472 & 182728 \\
\hline 1990 & 109132 & 102418 & 211550 \\
\hline 1999 & 108607 & 102533 & 211140 \\
\hline
\end{tabular}

* Effectifs par sexe non disponibles.

** En général, l'effectif total publié pour 1962 est basé sur les résultats exhaustifs, tandis que les effectifs estimés par sexe sont basés sur le sondage au quart.

Sources : Données de recensements de la population française publiées (INSEE, 1970 ; Marie, 1993 ; Marie et Rallu, 2004).

\section{Annexe 2 : Évolution du statut d'occupation des ménages dont la personne responsable (ou de référence) est née aux Caraïbes / aux Antilles françaises (en \%)}

2a. Répartition des ménages en Grande-Bretagne dont la personne responsable est née aux Caraïbes, selon le statut d'occupation

\begin{tabular}{|l|l|l|l|}
\hline Année & Propriétaires & Locataires d'HLM & Autres locataires \\
\hline 1966 & 41 & 7 & 52 \\
\hline 1971 & 44 & 21 & 35 \\
\hline 1981 & 43 & 45 & 12 \\
\hline
\end{tabular}

Source : adapté de Peach et Byron, 1993, p. 410. Les pourcentages entre parenthèses correspondent aux proportions pour l'ensemble des ménages britanniques.

2b. Pour des raisons de changement de catégorie de logement ou de regroupement des populations originaires des Dom-Tom d'un recensement à l'autre, il est impossible de fournir le même type de tableau pour le statut d'occupation des ménages « antillais » en France métropolitaine. À partir des données disponibles, il est possible de montrer l'évolution suivante pour les ménages dont la personne de référence est née aux Antilles :

\begin{tabular}{|l|l|l|l|l|l|}
\hline $\begin{array}{l}\text { Année du } \\
\text { recensement }\end{array}$ & Propriétaire & $\begin{array}{l}\text { Location } \\
\text { HLM }\end{array}$ & $\begin{array}{l}\text { Location vide secteur } \\
\text { privé }\end{array}$ & $\begin{array}{l}\text { Chambre } \\
\text { meublée }\end{array}$ & Autre \\
\hline \hline 1975 & 18 & $*$ & $*$ & 11 & 8 \\
\hline 1982 & 13 & 42 & 33 & 5 & 7 \\
\hline
\end{tabular}




\begin{tabular}{|l|l|l|l|l|l|l|}
\hline 1999 & 24 & 51 & 19 & 4 & 2 \\
\hline
\end{tabular}

Sources : INSEE, 1978 ; INSEE, 1985 ; INSEE, Recensement Général de la Population, France 1999 : Analyses de $\mathrm{S}$. Condon de données pour la population née aux Antilles à partir d'une exploitation particulière fournie par l'INSEE. La répartition des ménages résidant dans le secteur locatif en 1975 n'a pas été répartie selon le type de location.

Pour l'ensemble des ménages en France métropolitaine en 1999, la proportion de propriétaires était de $54 \%$, celle des locataires d'un logement social de $16 \%$ et les locataires du secteur privé comptaient pour 23 \% des ménages (INSEE, 1999, Recensement Général de la Population, France métropolitaine). Pour les ménages dont la personne de référence est née aux Antilles, comme pour l'ensemble des ménages, la proportion de locataires d'un HLM est plus élevée en région parisienne.

\section{Annexe 3}

3a. État matrimonial de la « minorité ethnique caribéenne » en Grande-Bretagne, 1991 ; sélection des groupes d'âges (en \%)

\begin{tabular}{|c|c|c|c|c|}
\hline Groupe « ethnique » & Célibataire & Marié/e & Divorcé/e & Veuf/veuve \\
\hline \multicolumn{5}{|l|}{ Femmes : } \\
\hline \multicolumn{5}{|l|}{ Blanches } \\
\hline $35-39$ & 10 & 77 & 12 & 1 \\
\hline $60-64$ & 6 & 70 & 6 & 18 \\
\hline \multicolumn{5}{|l|}{ Caribéennes } \\
\hline $35-39$ & 42 & 44 & 13 & 1 \\
\hline $60-64$ & 13 & 56 & 18 & 13 \\
\hline \multicolumn{5}{|l|}{ Hommes : } \\
\hline \multicolumn{5}{|l|}{ Blancs } \\
\hline $35-39$ & 17 & 73 & 9 & $<0,5$ \\
\hline $60-64$ & 9 & 80 & 7 & 4 \\
\hline \multicolumn{5}{|l|}{ Caribéens } \\
\hline $35-39$ & 36 & 54 & 10 & $<0,5$ \\
\hline $60-64$ & 13 & 68 & 14 & 5 \\
\hline
\end{tabular}

Source: OPCS, 1991 Census, England and Wales, Ethnic Group, Age and marital status, Table 6. 
3b. État matrimonial des femmes et des hommes âgés de 15 ans ou plus : comparaison des migrants antillais et de l'ensemble de la population en France métropolitaine, 1990 (en \%)

\begin{tabular}{|l|l|l|l|l|}
\hline & Célibataire & Marié/e & Divorcé/e & Veuf/veuve \\
\hline Ensemble des femmes & 28,2 & 52,9 & 5,2 & 13,7 \\
\hline $35-39$ & 14,2 & 75,5 & 9,1 & 1,2 \\
\hline $55-59^{*}$ & 7,0 & 75,1 & 6,0 & 11,9 \\
\hline Femmes antillaises & 50,1 & 39,4 & 7,4 & 3,1 \\
\hline $35-39$ & 34,6 & 54,5 & 10,3 & 0,6 \\
\hline $60-64$ & 21,6 & 49,7 & 15,1 & 13,6 \\
\hline Ensemble des hommes & 35,4 & 57,8 & 3,9 & 2,9 \\
\hline $35-39$ & 19,6 & 73,5 & 6,6 & 0,3 \\
\hline $55-59^{*}$ & 9,8 & 82,6 & 5,0 & 2,6 \\
\hline Hommes antillais & 48,3 & 46,0 & 4,9 & 0,8 \\
\hline $35-39$ & 30,8 & 62,8 & 6,3 & 0,1 \\
\hline $60-64$ & 11,6 & 73,3 & 11,1 & 4,0 \\
\hline
\end{tabular}

Les données publiées pour l'ensemble de la population métropolitaine au recensement de 1990 par état civil ne permettent pas de distinguer les 60-64 ans, mais seulement les 60-74 ans. Nous avons donc choisi le groupe des 55-59 ans pour la comparaison. Par ailleurs, les taux de divorce pour les 60-74 ans étaient de 4,4\% pour les femmes et de 3,5\% pour les hommes. Les résultats fournis pour la population antillaise ont été produits pour une publication séparée (Marie, 1993).

Sources : INSEE, 1992, Recensement de 1990. Population-Activité-Ménages. La France et ses régions, Table POP4DET p. 15 ; Marie, 1993.

\section{NOTES}

1. À titre d'exemple: Bougerol, 1998; Dagenais et Poirier, 1985 ; Ellis, 1986 ; Mulot, 2000 ; Pourette, 2006 ; Senior, 1991.

2. Les disparités entre le niveau de vie dans les îles antillaises et celui de la métropole n'étaient que trop manifestes, révélées clairement à travers les médias, la scolarisation, ainsi que dans les lettres ou les témoignages des parents ou amis émigrés en France. Tandis qu'en 1948, le régime de sécurité sociale (retraite pour les travailleurs âgés et remboursement des soins de santé) était introduit dans les DOM, le régime des prestations familiales (1946) n'était pas étendu aux DOM avant 1973 (son extension a été faite devant la montée des contestations politiques, mais il n'était pas aligné sur le régime métropolitain) et il a fallu attendre la fin des années 1970 pour qu'un ensemble de prestations concernant les personnes licenciées ou chômeuses soient introduites (Constant, 1987 : 11-15 ; voir aussi Gautier, 1993). 
3. Une littérature conséquente analyse le raisonnement fondant le choix de ne pas encourager la migration des Antillais vers la Grande-Bretagne, une politique motivée selon certains par des attitudes racistes à l'égard des habitants noirs des colonies (Carter et al., 1987 ; Fryer, 1984 ; Miles, 1984).

4. Enquête sur le fichier des migrants du Bumidom et entretiens auprès de migrants (Condon et Ogden, 1991). D'autres conscrits partaient vers la Guyane avec le service militaire adapté (SMA).

5. Dans le texte, «Just this?» (p. 21). Le roman d'Andrea Levy A small island décrit avec justesse et d'une manière vivante les conditions de vie des Londoniens au lendemain de la Seconde Guerre mondiale, ainsi que les espoirs et les attentes des hommes et des femmes qui avaient quitté les îles caribéennes pour s'installer à Londres à cette époque (Levy, 2004).

6. Pour des raisons historiques liées à l'urbanisation des deux capitales, à la différence de la France, dans les années 1960-80, la banlieue résidentielle du Grand Londres était recherchée par les classes moyennes. La inner city, la petite couronne autour du centre-ville de Londres était là où étaient construits les premiers grands ensembles de logement social.

7. Par exemple, l'enquête récente du PSI a révélé que $20 \%$ des Caribéens en couple avaient un partenaire d'un autre groupe ethnique (Berthoud et Beishon, 1997). Cette étude montre aussi, comme d'autres, que les hommes sont plus nombreux à se mettre en couple avec une femme d'un autre groupe que le sont les femmes; la même tendance a été retrouvée dans le cas français (Byron et Condon, 2008).

\section{RÉSUMÉS}

La famille caribéenne a longtemps fascinée les sociologues et les anthropologues nord-américains et britanniques. Malgré des références fréquentes aux liens forts entre les membres féminins de la parenté, le fait que ce "système familial», caractérisé par l'instabilité des couples soit diamétralement opposé au modèle de la famille patriarcale - reconnu comme la norme universelle de stabilité familiale et sociale - a conduit à ce que ces formes de famille soient considérées comme des structures inappropriées pour l'éducation des enfants.

Dans un contexte d'émigration et de circulation, la famille est actrice centrale; en même temps, face au processus migratoire et aux changements sociaux entraînés par la migration, la famille, les relations, les rôles évoluent. La spécificité supposée de la famille caribéenne sera explorée dans ce contexte, en analysant : l'installation des migrants en France métropolitaine ou au RoyaumeUni, les questions de résidence et l'évolution des formes familiales, de la «famille monoparentale " aux familles transnationales. La comparaison entre les contextes français et britannique des migrations caribéennes est éclairante sur les différents facteurs qui influencent les formes familiales et permet de nous éloigner des explications purement culturelles.

Migration, residence and representations of the family: Caribbean families in France and Britain. The "Caribbean family" has long intrigued North American and British family sociologists and anthropologists. Despite reference to cohesive bonds between female relatives, the divergence of this system, characterised by unstable unions, from the male-headed patriarchal family -held to be the universal norm of social and family stability- contributed to Caribbean family forms being considered as inadequate structures within which to bring up children.

In the context of emigration and circulation, the family plays a central role. At the same time, 
during the migration process and social changes induced by migration, family relationships and roles evolve. The assumed specificity of the "Caribbean family" will be explored within this context, through an analysis of both the settlement of migrants in metropolitan France and Britain, of issues relating to housing and residence and of the changes in family forms, from the single-parent family to transnational families. The comparison between the French and British contexts highlights the variety of factors influencing family forms and broadens the debate away from purely cultural explanations.

Migraciones, residencia y representaciones de la familia: las familias caribeñas en Francia y la Gran Bretaña. La familia caribena ha fascinado durante mucho tiempo a los sociólogos y a los antropólogos norteamericanos y británicos. A pesar de referencias frecuentes a los fuertes vínculos entre los miembros femeninos, el hecho de que este «sistema familiar», caracterizado por la inestabilidad de los pares sea opongado diametralmente al modelo de la familia patriarcal -reconocido como lo normalizó universal de estabilidad familiar y social- ha llegado a que estas formas de familia estén consideradas como estructuras inadecuadas para la educación de los niños.

En un contexto de emigración y circulación, la familia es actriz central; al mismo tiempo, ante el proceso migratorio y a los cambios sociales implicados por la migración, la familia, las relaciones, los papeles evolucionan. La especificidad supuesta de la familia caribena se explorará en este contexto, analizando: la instalación de los emigrantes en Francia metropolitana o en el Reino Unido, las cuestiones de residencia y la evolución de las formas familiares, de la «familia monoparental» a las familias transnacionales. La comparación entre los contextos franceses y británicos de las migraciones caribenas es luminosa sobre los distintos factores que influyen sobre las formas familiares y nos permite alejarnos de las explicaciones puramente culturales.

\section{INDEX}

Mots-clés : étude comparative, famille, Caribéens, parenté, relations familiales, Antillais

Index géographique : France, Royaume-Uni

\section{AUTEURS}

\section{STÉPHANIE CONDON}

Institut National d'Études Démographiques (INED), condon@ined.fr

\section{MARGARET BYRON}

Kings College, University of London, margaret.byron@kcl.ac.uk 